\title{
The National and Global Impact of Systemic and Structural Violence on the Effective Prevention, Treatment and Management of COVID-19 in the African/Black Population: a Scoping Review Protocol
}

Roberta Timothy ( $\nabla$ roberta.timothy@utoronto.ca )

University of Toronto, Dalla Lana School of Public Health https://orcid.org/0000-0002-2628-3235

\section{Robert Chin-see}

WLU: Wilfrid Laurier University

Julia Martyniuk

University of Toronto Gerstein Science Information Centre

\section{Djiadeu Pascal}

McMaster University Department of Health Research Methods Evidence and Impact https://orcid.org/0000-0001-9708-6530

\section{Protocol}

Keywords: African, Blacks, COVID-19, Systemic/structural Barriers, health disparities, minority health, racial health inequity, structural violence, anti-Black racism, decolonizing, resistance, social justice

Posted Date: November 15th, 2021

DOI: https://doi.org/10.21203/rs.3.rs-1029118/v1

License: (c) (1) This work is licensed under a Creative Commons Attribution 4.0 International License. Read Full License 


\section{Abstract}

Background: As the SARS-CoV-2 virus continues to ravage the globe and cases exploded rapidly, countries have been presented with challenging policy choices to contain the spread of COVID-19. In Canada, and globally, the COVID-19 pandemic has added a new stratum to the debate concerning the root causes of global and racial health inequities and disparities. Individuals who exist as targets of systemic inequities are not only more susceptible to contracting COVID-19, but they are also more likely to bear the greatest extent of the subsequent economic pandemic. Therefore, data collection that specifically focuses on the impact of COVID-19 on the lives and health of African/Black communities nationally and globally is needed to develop intersectional, culturally-relative, anti-racist/anti-oppression, empowermentcentered interventions and social policies to increase more efficient ways to support heterogeneous African/Black communities during and after the COVID-19 pandemic.

The primary objective of this review is to investigate the impact and management of COVID-19 on African/Black individuals and communities in Canada and globally and understand how anti-black racism and intersectional violence impact the health of African/Black communities during the COVID-19 pandemic.

Methods and analysis: A systematic search of published literature of quantitative and qualitative studies published on COVID-19 in Canada and globally will be conducted in Ovid Medline, Ovid EMBASE, EBSCO Cumulative Index to Nursing and Allied Health Literature, the Cochrane Library, Ovid Psychlnfo, Ovid CAB Abstracts, Scopus, Web of Science, and Global Index Medicus.

To be included in the review, studies should include data on COVID-19 in relation to African/Black individuals, population and communities in Canada and globally. The studies must discuss racism, oppression, anti-oppression, or systemic/structural violence and be published in English, French, Spanish, and Portuguese. The findings will be reported according to PRISMA-ScR.

Conclusion: This review will collect important data and evidence on African/Black communities related to COVID-19. Moreover, this review could help identified existing gaps in COVID-19 management in the African/Black communities and inform future research. Furthermore, it could also be used in decisionmaking for health policy and promotion and can influence the services provided by healthcare facilities and community organizations around the globe.

Systematic Review registrations: Open Science Framework (OSF). Submitted on November 1st, 2021.

\section{Background}

As the SARS-CoV-2 virus continues to ravage the globe and cases exploded rapidly, countries have been presented with challenging policy choices to contain the spread of COVID-19. Despite this representing a global effort; each country had a different response to the pandemic. For example, some countries such as China and South Africa elected to immediately close the national economy and apply strict and 
punishable rules on traditional public health measures (e.g., social distancing, masking, isolation and quarantine) ${ }^{1}$. In contrast, others countries, including the United States, opted for more loose public health recommendations. Although research has found that public health interventions and non-pharmaceutical control measures have been effective in mitigating transmission of COVID-19, the differential timing of lockdown measures, including closing non-essential industries and limiting in-person capacity, may have significant social and economic implications ${ }^{2,3}$. One of the potential concerns is how the COVID-19 pandemic has differentially affected populations as it appears that the burden was not borne equal. The COVID-19 pandemic has created a wave of panic across the world. According to John Hopkins University, COVID-19 has taken the life of 4,310,354 people across the globe with 26,633 deaths in Canada alone, as of August 10th $2021^{4}$. The COVID-19 pandemic has added a new stratum to the debate concerning the root causes of racial health disparities. The effects of COVID-19 has been shown to be linked to stuctural violence and/or racism ${ }^{5}$. The British and American governments have acknowledged that a large proportion of their COVID-19 patients and deaths are from African descent. Due to the discrimination and oppression suffered by racialized groups such as black communities, it is reported that in England, Black people were more than 4 times likely to die from the corona virus than their white counterparts ${ }^{5,6}$. Similarly, an analysis by the Washington Post reports that in the United States, counties with Black majorities have three times the rate of covid-19 cases, and almost six times the rate of deaths, compared with counties where white residents are in the majority ${ }^{7}$.

Multiple researchers have misattributed the morbidity and mortality disparities observed in England and America to the high prevalence of chronic disease in Black communities ${ }^{8,9}$. Multinational data has reported poor outcomes for individuals over 65 , and/or with underlying health conditions, including diabetes, heart disease, asthma, and compromised immune systems ${ }^{10}$.

The association between COVID-19 and pre-existing illness is especially troubling for Black individuals who are genetically misconceived as more likely to develop chronic comorbidities because they are Black 11.

Many social determinants of health-including Anti-Black racism, intersectional violence (including sexism, heterosexism, classism, ageism, refugee status, among others), poverty, physical environment (e.g., smoke exposure, homelessness), and race and ethnicity - can have a considerable effect on COVID19 outcomes. Homeless families are at higher risk of viral transmission because of crowded living spaces and scarce access to COVID-19 screening and testing facilities ${ }^{12}$.

Individuals who exist as targets of systemic inequities are not only more susceptible to contracting COVID-19, but they are also more likely to bear the greatest extent of the subsequent economic pandemic 13.

There is a need for data collection that specifically focuses on the impact of COVID-19 on the lives and health of African/Black communities nationally and globally in order to develop intersectional, culturallyrelative, anti-racist/anti-oppression, empowerment-centered interventions and social policies that increase 
more efficient ways to support heterogeneous African/Black communities during and after the COVID-19 pandemic.

In Canada and globally, the collection of race based data on COVID-19 is important to understand the impact of COVID-19 on the lives of African/Black people and its historical and current day context ${ }^{14}$.

In most African countries, the response to the COVID-19 pandemic has been challenging due to continued colonial impacts, which lead to distrust in government, social, cultural and religious resistance ${ }^{15}$. The COVID-19 global pandemic has exposed the world inequities and the racially based colonial demarcations with the North/South as main geographical and sociological anchors ${ }^{16}$. In Brazil, the failure of the neoliberal government to protect the Black and Indigenous populations mostly exposed to COVID-19 infection, has created the emergence of a new form of solidarity and mutual aid in "favelas" and Indigenous communities ${ }^{17}$.

The purpose of this scoping review is to employ a decolonizing, African feminist, Black resistance lens to examine the impact of COVID-19 on heterogeneous and intersectional Black communities in Canada and throughout the world; while also exploring the various forms of resistance that Black communities have established and employed during the global pandemic. Therefore, this review will attempt to answer the question as to how COVID-19 is impacting African/Black communities nationally and globally and what interventions are effective to prevent, treat, and reduce the impact of COVID-19 on these communities?

The primary objective is to: 1 ) investigate the impact of COVID-19 on African/Black individuals, communities, front line health care workers, and essential service workers; 2)explore how systemic/structural violence are barriers to effective prevention, treatment and management of COVID-19 in the African/Black population; and 3)understand how anti-black racism and intersectional violence (violence related to race, gender, sexual orientation, gender identity, age, disabilities, language, educational attainment, immigration status and social determinants of health) impact the health of African/Black communities during the COVID-19 pandemic. The secondary objective of the review is to identify intervention strategies to respond effectively to the impact of COVID-19 on African/Black individuals, communities, front line health service workers, and essential service workers in Canada and transnationally.

Our primary outcomes are the impact of COVID-19 on African/Black individuals, front line health care workers and essential service workers, the effect of systemic and structural barriers on prevention and treatment management on these communities and the effect of social determinants of health (SDOH) as well as the intersection of gender, sexual orientation, age, disabilities, language, immigration status, among other intersectional factors, on the COVID-19 pandemic in these communities.

\section{Methods And Analyses}


A scoping review methodology was selected as it (1) helped to identify review parameters, (2) identified a process of mapping the existing literature, and (3) explored a research gap ${ }^{18}$.

The methodological framework as described by Arksey and O'Malley and later advanced by Levac et al will be applied and followed in developing and disseminating this scoping review ${ }^{19,20}$. The scoping review approach will also endeavour to include recommendations by Peterson that is appropriate for policy change, education and research purposes ${ }^{21}$. The protocol was drafted with intention to align with the reporting guidance provided in the Preferred Reporting Items for Systematic Reviews and MetaAnalyses Protocols (PRISMA-P) statement and the Preferred Reporting Items for Systematic Reviews and Meta-Analyses extension for Scoping Reviews (PRISMA-ScR)-Appendix 12-24. This framework $^{22}$ recommends organizing the scoping review in a five-stage process which includes but is not limited to: (1) identifying the research question; (2) identifying relevant studies; (3) study selection; (4) charting the data; (5) collating, summarising and reporting the results; and (6) consulting with relevant stakeholders and key informants.

To be clear, standard systematic reviews and scoping reviews are different; scoping reviews investigate broad topics as opposed to a specific well-defined question. In the context of this paper, this review will assess the impact of COVID-19 on African/Black community in Canada and across the globe.

\section{Criteria for including studies}

\section{Types of studies}

Experimental (randomised or non-randomised), observational (Iongitudinal, cross-sectional), qualitative, and mixed methods studies will be considered for this review.

\section{Inclusion and Exclusion Criteria}

To be included in the review, studies should include data on COVID-19 in relation to African/Black individuals, population and communities in Canada and globally. The studies must discuss racism, oppression, anti-oppression, or systemic/structural violence and be published in English, French, Spanish, and Portuguese. Screening will be conducted to filter out studies based on to their publication date and language. In particular, studies performed before December 2019 will be excluded.

\section{Inclusion criteria:}

- Must discuss COVID-19

- Must discuss African/Black individuals and communities in Canada and globally

- Must be in English, French, Spanish or Portuguese

- Must discuss racism, oppression, anti-oppression, or systemic or structural violence

\section{Exclusion criteria:}


- Review papers (scoping, systematic), reports, book's chapters and conference abstract will be excluded from the review.

\section{Outcomes}

The Primary outcomes of this scoping review will be to:

Evaluate the impact of COVID-19 on African/Black individuals, communities, health care workers and essential service workers across Canada and globally.

- Investigate the effect of systemic and structural barriers on prevention, treatment and management of COVID-19 in a population that has been reported to experience significantly higher COVID-19 complications and negative outcomes

- Evaluate the impact of social determinants of health and the intersections of dimensions such as gender, sexual orientation, gender identity, age, disabilities, language, educational attainment, immigration status, on COVID-19 in African/Black communities.

\section{Secondary outcomes}

We will assess:

- The resistance of African/Black communities in relations to the structural barriers that they face in the context of COVID-19.

- The population-based intervention strategies and tools to better prevent, treat, and manage COVID-19 in African/Black individuals, communities, front line health care workers and essential service workers in Canada and globally

\section{Electronic searches}

A comprehensive literature search will be conducted in Ovid Medline, Ovid EMBASE, EBSCO Cumulative Index to Nursing and Allied Health Literature (CINAHL), the Cochrane Library, Ovid PsychInfo, Ovid CAB Abstracts, Scopus, Web of Science and Global Index Medicus. Electronic search strategies will be developed by the health science librarian (JM) and will be peer-reviewed according to the peer review of electronic search strategies (PRESS) guidelines ${ }^{25}$. The literature search will systematically search the published literature of quantitative and qualitative studies published since the beginning of COVID-19 in December 2019, and will be documented in accordance with the PRISMA-ScR checklist ${ }^{23}$.

The search strategy can be found in Box 1 and include the concepts Covid-19, Black people, and racism.

Box 1: Proposed Search Strategies for Medline (Search Ran Aug 4, 2021). 
1 exp Coronavirus/

87181

2 exp Coronavirus Infections/

106533

3 (coronavirus* or corona virus* or OC43 or NL63 or 229E or HKU1 or HCoV* or ncov*

173610 or covid* or sars-cov* or sarscov* or Sars\$coronavirus* or Severe Acute Respiratory

Syndrome Coronavirus* or $2019 \$$ nCov or Severe Acute Respiratory Syndrome Corona Virus).tw,kf,ot.

4 ((novel or new or nouveau) adj2 (CoV or nCoV or covid* or coronavirus* or corona virus or Pandemi*)).tw,kf,ot.

5 ((Wuhan or Hubei) adj5 pneumonia).tw,kf,ot.

6 ((new or novel or "19" or "2019" or Wuhan or Hubei or China or Chinese) adj3

(coronavirus* or corona virus* or betacoronavirus* or CoV or HCoV)).tw,kf,ot.

7 ((coronavirus* or corona virus* or betacoronavirus*) adj3 (pandemic* or epidemic* or outbreak* or crisis)).tw,kf,ot.

81 or 2 or 3 or 4 or 5 or 6 or 7

9 limit 8 to $y r=" 2019$-Current"

10 exp african continental ancestry group/ or ethnic groups/

11 Minority Groups/

12 Minority Health/

13 (people of colo\$̣r or person* of colo\$r or POC or BAME or BIPOC or ((african* or afro*) adj5 (americ* or canad* or asia* or caribbean* or australi* or european* or brazil* or minorit* or refugee or migrant* or immigrant* or ancest* or native* or hispanic* or latin* or indigenous* or diaspora* or communit* or descen* or provider* or nurse* or doctor* or worker* or service user* or patient* or front line* or frontline* or people* or man or men or wom\$n or race or population* or person* or individual* or group* or female* or male*))).tw,kf.

14 ((black or blacks) adj5 (americ* or canad* or asia* or caribbean* or australi* or european* or brazil* or minorit* or refugee or migrant* or immigrant* or ancest* or native* or hispanic* or latin* or indigenous* or diaspora* or communit* or descen* or provider* or nurse* or doctor* or worker* or service user* or patient* or front line* or frontline* or people* or man or men or wom $\$ n$ or race or population* or person* or individual* or group* or female* or male*)).tw,kf.

15 (((ethnic* or racial* or race) adj5 (group* or minorit* or disparit* or divers* or equal* or inequal* or discriminat*) ) or mixed race or mixed racial* or multi racial* or mutli race or multiracial* or multirace).tw,kf.

1610 or 11 or 12 or 13 or 14 or 15

17 prejudice/ or racism/

18 (racism or racist* or racial* or anti-black* or antiblack* or structural violence* or systemic violence*).tw,kf. 


\begin{tabular}{|c|c|c|}
\hline \# & Searches & Results \\
\hline 19 & (white supremac* or white hegemon*).tw,kf. & 95 \\
\hline 20 & (prejudice* or discriminat* or intolerance* or oppress* or bias* or hostil*).tw,kf. & 550272 \\
\hline 21 & $\begin{array}{l}\left(\left(\text { structur* or institution* }{ }^{\star} \text { or systemic or systematic* or generational* or intersect* or }\right.\right. \\
\text { health*) adj5 (violence* or polic* or barrier* or disparit* or inequalit* or } \\
\text { trauma)).tw,kf. }\end{array}$ & 145097 \\
\hline 22 & 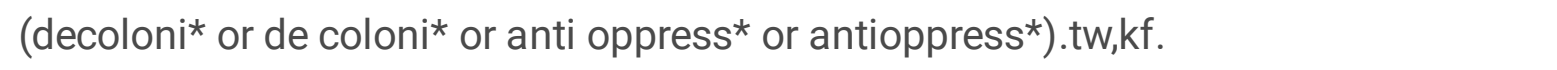 & 1798 \\
\hline 23 & 17 or 18 or 19 or 20 or 21 or 22 & 744913 \\
\hline 24 & 9 and 16 and 23 & 1349 \\
\hline
\end{tabular}

\section{Reference lists}

The reference lists of all relevant citations will be searched for available related articles.

\section{Grey literature}

We will search for available theses and conference posters. Furthermore, experts, authors and relevant organization will be contacted.

\section{Study Selection Process/Screening}

Search results will be de-duplicated in Endnote before being uploaded to Covidence (JM), an online program that facilitates screening and data extraction ${ }^{26}$.

Two reviewers will pilot test a screening form customized to reflect the aforementioned inclusion criteria. This screening form will be generated and used by two independent reviewers. A subset of record will be used as a sample to establish consistency of use and clarity of instrument, before its implementation. A Cohen's kappa statistic will be estimated to measure inter-rater reliability and screening will begin when $90 \%$ agreement is achieved ${ }^{27}$.

The following describes the study selection: To start, we will conduct a title and abstract screening. Once an article is seen as potentially relevant, we will retrieve and screen the full text in detail. This occurs prior to data extraction. In duplicate, (RCS and PD) will conduct all screening, data extraction and quality assessment. Disagreements will be resolved by consensus. If consensus cannot be reached, a third author (RT) will arbitrate.

\section{Data extraction}

For the purpose of this scoping review, we will extract bibliometric information such as author names, journal, and year of publication, in addition to the location of the study, study design, number of 
participants, outcomes reported, outcome measures. We will report where possible measures of effect of the outcome on African/Black with respect to COVID-19. We will not, report measures of magnitude mean (standard deviation) or percent (95\% confidence intervals), or extract data such as odds or risk ratios, mean differences.

\section{Data Analyses and reporting}

Our findings will be reported according to the PRISMA-ScR guidelines ${ }^{23,28,29}$. Our findings will be summarized narratively and using tables. Data will be grouped by outcomes, with the number of studies, their design and their methodological quality. Key findings of each study will also be summarized using tables. We will conduct a narrative synthesis of the data to identify common themes and knowledge gaps.

\section{Discussion}

The global corona virus pandemic is still occurring more than a year after its initial emergence in late December 2019. Published studies highlight the inequity and disparity in corona virus infections, and deaths. Data collected globally as well as research indicate that African and Black populations are more likely to contract and die from COVID-19 5,6,9,30.

Anti- Black racism and colonial institutions contribute to disparities in access to health care, employment, education, housing, physical environments, and food insecurity ${ }^{31,32}$. These determinants have been shown to have an impact on corona virus infection ${ }^{14,16,33}$. Through this scoping review, we will employ a decolonizing, African feminist, Black resistance lens to investigate the impact of COVID-19 on heterogeneous and intersectional Black communities in Canada and throughout the world. We will also explore the various forms of resistance that Black communities have established and employed during the global COVID-19 pandemic.

The exhaustive search strategy across numerous global databases is one of the strengths of this review. Another strength is the innovative research topic that examines the global impact of COVID-19 on African/Black communities around the world.

One limitation of this scoping review is the wide range of the inclusion criteria. Thus, the hand-searching of the literature by the research team could lead to personal interpretation of the criteria. Furthermore, the search was not translated into the four inclusion languages, and studies could be missed because of this. Also, it is generally understood that scoping reviews are not intended to be exhaustive, especially compared to systematic reviews and meta-analyses ${ }^{34}$. Still, the lack of critical appraisal, or possibility in missing and evaluating relevant studies has been reported as a key challenge in conducting scoping reviews ${ }^{35,36}$. However, a narrative synthesis was found appropriate which is both widely used and recommended in guidelines for scoping reviews. Another limitation to consider is the differences and nuances in terminology across languages and regions. For example, systemic and structural racism may 
be described differently in different native languages using different terms. This may lead to a bias due to the lack of sufficiently locally published articles in non-English journals in this review.

\section{Conclusions}

This review will collect important data and evidence on African/Black communities related to COVID-19. Most importantly this review could be used in decision-making for health policy and promotion and can influence the services provided by healthcare facilities and community organizations around the globe that serve individuals from African and Black communities and help mitigate COVID-19 risk and ameliorate health outcomes and trajectories.

\section{Abbreviations}

SARS-CoV-2: Severe acute respiratory syndrome coronavirus 2; COVID-19: Coronavirus disease 2019; SDOH: Social Determinants of Health; PRISMA-R: Preferred Reporting Items for Systematic Reviews and Meta-Analyses Protocols; PRISMA-ScR: Preferred Reporting Items for Systematic Reviews and MetaAnalyses extension for Scoping Reviews (PRISMA-ScR); EMBASE: Excerpta Medica Database; EBSCO: Elton Bryson Stephens Sr. Company; CINAHL: Cumulative Index to Nursing and Allied Health Literature

\section{Declarations}

\section{Ethics and dissemination:}

Ethics approval is not required as secondary published data will be used. Our findings will be disseminated as peer reviewed manuscripts, at conferences, student rounds and could be of interest to government health agencies and organizations serving the African/Black communities.

\section{Patients and Public Involvement:}

Patients will not directly be involved in this study.

\section{Consent for publication:}

Not applicable

\section{Availability of data and material:}

All data generated or analysed during this study will be included in the published article and its supplementary information files.

\section{Competing interests' statement:}

The authors declare that they have no competing interests. 


\section{Funding statement:}

This paper was made possible through the Institute for Pandemics - Dalla Lana School of Public Health, University of Toronto and the Balsam Foundation in Toronto.

\section{Author contributions:}

The study was conceived by RT. All authors revised the research question and provided content to the design. Manuscript was written and edited by RT, PD, RCS and JM. Principal investigator of the study is RT. All authors read and approved the final version of the manuscript.

\section{Acknowledgment:}

Not applicable

\section{References}

1. Arndt C, Davies R, Gabriel S, et al. Covid-19 lockdowns, income distribution, and food security: An analysis for South Africa. Global Food Security. 2020;26:100410.

2. Ayouni I, Maatoug J, Dhouib W, et al. Effective public health measures to mitigate the spread of COVID-19: a systematic review. BMC public health. 2021;21(1):1-14.

3. Coibion O, Gorodnichenko Y, Weber M. The cost of the covid-19 crisis: Lockdowns, macroeconomic expectations, and consumer spending. National Bureau of Economic Research;2020.

4. Coronavirus Resource Center. 2021. https://coronavirus.jhu.edu/map.html. Accessed August 10th, 2021.

5. Khunti K, Singh AK, Pareek M, Hanif W. Is ethnicity linked to incidence or outcomes of covid-19? : British Medical Journal Publishing Group; 2020.

6. Patel P, Hiam L, Sowemimo A, Devakumar D, McKee M. Ethnicity and covid-19. British Medical Journal Publishing Group; 2020.

7. Thebault $R$, Tran $A B$, Williams $V$. The coronavirus is infecting and killing black Americans at an alarmingly high rate. Washington Post. 2020;7.

8. Williamson E, Walker AJ, Bhaskaran K, et al. OpenSAFELY: factors associated with COVID-19-related hospital death in the linked electronic health records of 17 million adult NHS patients. MedRxiv. 2020.

9. Aldridge RW, Lewer D, Katikireddi SV, et al. Black, Asian and Minority Ethnic groups in England are at increased risk of death from COVID-19: indirect standardisation of NHS mortality data. Wellcome open research. 2020;5.

10. Organization WH. Coronavirus disease 2019 (COVID-19): situation report, 86. 2020.

11. Etowa J, Hyman I. Unpacking the health and social consequences of COVID-19 through a race, migration and gender lens. Canadian Journal of Public Health. 2021;112(1):8-11. 
12. Abrams EM, Szefler SJ. COVID-19 and the impact of social determinants of health. The Lancet Respiratory Medicine. 2020;8(7):659-661.

13. Ruiz NG, Horowitz JM, Tamir C. Many Black and Asian Americans say they have experienced discrimination amid the COVID-19 outbreak. Pew Research Center. 2020;1.

14. University JH. Racial Data Transparency-States that have released breakdowns of Covid-19 data by race. 2020.

15. Adebisi YA, Rabe A, Lucero-Prisno III DE. Risk communication and community engagement strategies for COVID-19 in 13 African countries. Health Promotion Perspectives. 2021;11(2):137-147.

16. Oliveira RGd, Cunha APd, Gadelha AGdS, Carpio CG, Oliveira RBd, Corrêa RM. Racial inequalities and death on the horizon: COVID-19 and structural racism. Cadernos de saude publica. 2020;36:e00150120.

17. Ortega F, Orsini M. Governing COVID-19 without government in Brazil: Ignorance, neoliberal authoritarianism, and the collapse of public health leadership. Global public health. 2020;15(9):12571277.

18. Pham MT, Rajić A, Greig JD, Sargeant JM, Papadopoulos A, McEwen SA. A scoping review of scoping reviews: advancing the approach and enhancing the consistency. Research synthesis methods. 2014;5(4):371-385.

19. Arksey H, O'Malley L. Scoping studies: towards a methodological framework. International journal of social research methodology. 2005;8(1):19-32.

20. Levac D, Colquhoun H, O'Brien KK. Scoping studies: advancing the methodology. Implementation science. 2010;5(1):1-9.

21. Peterson J, Pearce PF, Ferguson LA, Langford CA. Understanding scoping reviews: Definition, purpose, and process. Journal of the American Association of Nurse Practitioners. 2017;29(1):12-16.

22. Sarkis-Onofre R, Catalá-López F, Aromataris E, Lockwood C. How to properly use the PRISMA Statement. Systematic Reviews. 2021;10(1):1-3.

23. Tricco AC, Lillie E, Zarin W, et al. PRISMA extension for scoping reviews (PRISMA-ScR): checklist and explanation. Annals of internal medicine. 2018;169(7):467-473.

24. Moher D, Shamseer L, Clarke M, et al. Preferred reporting items for systematic review and metaanalysis protocols (PRISMA-P) 2015 statement. Systematic reviews. 2015;4(1):1-9.

25. McGowan J, Sampson M, Salzwedel DM, Cogo E, Foerster V, Lefebvre C. PRESS peer review of electronic search strategies: 2015 guideline statement. Journal of clinical epidemiology. 2016;75:4046.

26. Covidence. Covidence systematic review software, Veritas Health Innovation, Melbourne, Australia.

27. Viera AJ, Garrett JM. Understanding interobserver agreement: the kappa statistic. Fam med. 2005;37(5):360-363.

28. Bernardo WM. PRISMA statement and PROSPERO. International braz j urol. 2017;43(3):383-384. 
29. Stroup DF, Berlin JA, Morton SC, et al. Meta-analysis of observational studies in epidemiology: a proposal for reporting. Jama. 2000;283(15):2008-2012.

30. Phiri P, Delanerolle G, Al-Sudani A, Rathod S. COVID-19 and black, Asian, and minority ethnic communities: a complex relationship without just cause. JMIR public health and surveillance. 2021;7(2):e22581.

31. Williams DR, Mohammed SA. Racism and health II: a needed research agenda for effective interventions. American behavioral scientist. 2013;57(8):1200-1226.

32. Czyzewski K. Colonialism as a broader social determinant of health. International Indigenous Policy Journal. 2011;2(1).

33. Kopel J, Perisetti A, Roghani A, Aziz M, Gajendran M, Goyal H. Racial and gender-based differences in COVID-19. Frontiers in public health. 2020;8:418.

34. Levett-Jones T, Hoffman K, Dempsey J, et al. The 'five rights' of clinical reasoning: An educational model to enhance nursing students' ability to identify and manage clinically 'at risk'patients. Nurse education today. 2010;30(6):515-520.

35. Hasan M, Singh H, Haffizulla F. Culturally Sensitive Health Education in the Caribbean Diaspora: A Scoping Review. International Journal of Environmental Research and Public Health. 2021;18(4):1476.

36. Salami B, Fernandez-Sanchez H, Fouche C, et al. A Scoping Review of the Health of African Immigrant and Refugee Children. International journal of environmental research and public health. 2021;18(7):3514.

\section{Supplementary Files}

This is a list of supplementary files associated with this preprint. Click to download.

- Appendix1BHMPRISMAScRChecklistFinalSYSR10262021.pdf 